PROCEEDINGS OF THE

AMERICAN MATHEMATICAL SOCIETY

Volume 131, Number 2, Pages 389-399

S 0002-9939(02)06836-3

Article electronically published on September 25, 2002

\title{
DIFFERENTIABILITY AND REGULARITY OF LIPSCHITZIAN MAPPINGS
}

\author{
BORIS S. MORDUKHOVICH AND BINGWU WANG
}

(Communicated by Jonathan M. Borwein)

\begin{abstract}
AвSTRACT. We introduce new differentiability properties of functions between Banach spaces and establish their relationships with graphical regularity of Lipschitzian single-valued and set-valued mappings. The proofs are based on advanced tools of nonsmooth variational analysis including new results on coderivative scalarization and normal cone calculus.
\end{abstract}

\section{INTRODUCTION}

Lipschitzian properties of single-valued and set-valued mappings play a crucial role in many aspects of nonsmooth variational analysis; see [10. It follows from the results of Rockafellar [9] that for every function $f: \mathbb{R}^{n} \rightarrow \mathbb{R}^{m}$ locally Lipschitzian around $\bar{x}$ Clarke's tangent cone to the graph of $f$ at $(\bar{x}, f(\bar{x}))$ is a linear subspace of dimension $d \leq n$ in $\mathbb{R}^{n} \times \mathbb{R}^{m}$, where $d=n$ if and only if $f$ is strictly differentiable at $\bar{x}$. This implies that nonsmooth Lipschitzian mappings cannot exhibit graphical regularity, i.e., Clarke's tangent cone to their graphs never agrees with Bouligand's contingent cone at reference points.

The primary goal of this paper is to establish dual counterparts of these and related results for mappings between infinite-dimensional spaces; see Section 4 . We show, in particular, that the subspace property holds for Clarke's normal cone to graphs of compactly Lipschitzian mappings (in the sense of Thibault) and obtain other results in this direction. To establish differential characterizations of graphical regularity in infinite dimensions, we introduce and study new notions of "weak $\beta$ differentiability" and "weak strict $\beta$-differentiability" (with respect to any given bornology $\beta$ ) that may be weaker than even Gâteaux differentiability for some Lipschitzian mappings $f: \mathbb{R} \rightarrow \ell^{2}$; see Section 2 . We also extend these results to the case of sets and set-valued mappings whose graphs are generated by graphs of single-valued Lipschitzian mappings via smooth transformations with surjective derivatives; see Section 5.

Our main tools relate to the analysis of nonconvex limiting normal cones to sets and the corresponding coderivatives of mappings. We consider these objects

Received by the editors March 23, 2001.

2000 Mathematics Subject Classification. Primary 49J52; Secondary 58C20.

Key words and phrases. Lipschitzian mappings, differentiability, Banach spaces, variational analysis, graphical regularity.

The first author was partly supported by the National Science Foundation under grants DMS9704751 and DMS-0072179 and also by the Distinguished Faculty Fellowship at Wayne State University. 
in Section 3 using arbitrary linear topologies on dual spaces that lie between the weak-star and norm topologies. This way allows us to unify some known facts and to obtain new ones, particularly on coderivative scalarization, important for establishing the main results of the paper.

Throughout the paper we use standard notation. Unless otherwise stated, all the spaces under consideration are Banach, and their norms are denoted by $\|\cdot\|$. Given a space $X, \mathbb{B}_{X}$ stands for its closed unit ball.

\section{Differentiability}

Recall that a bornology $\beta$ on $X$ is a family of bounded and centrally symmetric subsets of $X$ whose union is $X$, which is closed under multiplication by positive numbers and such that the union of any two members of $\beta$ is contained in some member of $\beta$. We identify three particular bornologies: the Gâteaux bornology, $\beta=G$, consisting of all finite symmetric sets (the weakest one); the Hadamard bornology, $\beta=H$, consisting of all symmetric sets compact in the norm topology; and the Fréchet bornology, $\beta=F$, consisting of all bounded symmetric sets (the strongest one).

Every bornology generates a certain concept of differentiability. A mapping $f: X \rightarrow Y$ is strictly $\beta$-differentiable at $\bar{x}$ if there is a bounded linear operator $A:=\nabla_{s \beta} f(\bar{x}): X \rightarrow Y$ such that

$$
\lim _{x \rightarrow \bar{x}, t \downarrow 0}\|[f(x+t v)-f(x)] / t-A v\|=0 \text { for all } v \in X,
$$

where the convergence is uniform with respect to $v$ in each set of $\beta$. When $x=\bar{x}$ in (1), $f$ is said to be $\beta$-differentiable at $\bar{x}$ with $\beta$-derivative $A:=\nabla_{\beta} f(\bar{x})$.

Now let us introduce "weak" counterparts of these notions and show that they may be of independent interest. Given $y^{*} \in Y^{*}$, we define a real-valued function $\left\langle y^{*}, f\right\rangle: X \rightarrow \mathbb{R}$ by $\left\langle y^{*}, f\right\rangle(x):=\left\langle y^{*}, f(x)\right\rangle$ for all $x \in X$.

Definition 2.1. (i) A mapping $f: X \rightarrow Y$ is WEAKLY STRICTLY $\beta$-DIFFEREnTIABLE (abbreviated by $w s \beta$-differentiable) at $\bar{x}$ if the scalarized function $\left\langle y^{*}, f\right\rangle$ is strictly $\beta$-differentiable at $\bar{x}$ for all $y^{*} \in Y^{*}$. We say that $f$ admits a ws $\beta$ DERIVATIVE at $\bar{x}$ if there is a bounded linear operator $A:=\nabla_{w s \beta} f(\bar{x}): X \rightarrow Y$ such that

$$
\lim _{x \rightarrow \bar{x}, t \downarrow 0}\left\langle y^{*},[f(x+t v)-f(x)] / t-A v\right\rangle=0 \text { for all } v \in X, y^{*} \in Y^{*},
$$

where the convergence is uniform with respect to $v$ in each set of $\beta$.

(ii) $f$ is WEAKLY $\beta$-DIFFERENTIABLE (abbreviated by $w \beta$-differentiable) at $\bar{x}$ if $\left\langle y^{*}, f\right\rangle$ is $\beta$-differentiable at $\bar{x}$ for all $y^{*} \in Y^{*}$. If (2) holds with $x=\bar{x}$, the operator $A:=\nabla_{w \beta} f(\bar{x})$ is called the $w \beta$-DERIVATIVE of $f$ at $\bar{x}$.

The terminology comes from the fact that the weak convergence on $Y$ is used in (2) instead of the norm one used in (1). Observe that the $w s \beta$-derivatives and the $w \beta$-derivatives are unique when they exist, and that the corresponding notions in (11) and Definition 2.1 agree if $\operatorname{dim} Y<\infty$. The following example shows that it is no longer the case if $\operatorname{dim} Y=\infty$ : a Lipschitzian mapping may be weakly strictly differentiable with respect to the strongest Fréchet bornology but not even Gâteaux differentiable!

Example 2.2. There is a Lipschitz continuous mapping $f: \mathbb{R} \rightarrow \ell^{2}$ which is $w s F$ differentiable at $\bar{x}=0$ but not $G$-differentiable at this point. 
Proof. Let $\psi: \mathbb{R} \rightarrow \mathbb{R}$ be a $C^{\infty}$-smooth function such that $\psi \neq$ const, $\operatorname{supp}(\psi) \subset$ $(0,1)$, and both $\psi$ and $\nabla \psi$ are bounded by some $M>0$. Consider a complete orthonormal basis $\left\{e_{1}, e_{2}, \ldots\right\}$ in the Hilbert space $\ell^{2}$ and define $f(x):=$ $\sum_{k=1}^{\infty} \psi_{k}(x) e_{k}$ with $\psi_{k}(x):=2^{-k} \psi\left(2^{k}\left(x-2^{-k}\right)\right)$ for all $x \in \mathbb{R}$. For each $k, j \in \mathbb{N}$ with $k \neq j$ we have $\operatorname{supp}\left(\psi_{k}\right) \cap \operatorname{supp}\left(\psi_{j}\right)=\emptyset$. Therefore for any $x \in \mathbb{R}, \psi_{k}(x) \neq 0$ for at most one $k \in \mathbb{N}$. This implies the Lipschitz continuity of $f$ on $\mathbb{R}$. Define $g(x):=\left\langle y^{*}, f\right\rangle(x)=\sum_{k=1}^{\infty} y_{k} \psi_{k}(x)$ for $y^{*} \in \ell^{2}$, where $y_{k} \in \mathbb{R}$ are uniquely determined by the representation $y^{*}=\sum y_{k} e_{k}$. Then

$$
\left|g\left(x_{1}\right)-g\left(x_{2}\right)\right|=\left|y_{k_{1}} \psi_{k_{1}}\left(x_{1}\right)-y_{k_{2}} \psi_{k_{2}}\left(x_{2}\right)\right| \leq\left(\left|y_{k_{1}}\right|+\left|y_{k_{2}}\right|\right) M\left|x_{1}-x_{2}\right|,
$$

where $k_{i} \geq \log _{2} \eta^{-1}$ if $\left|x_{i}\right|<\eta, i=1,2$. This yields $g\left(x_{1}\right)-g\left(x_{2}\right)=o\left(\left|x_{1}-x_{2}\right|\right)$ as $x_{1}, x_{2} \rightarrow 0$, which proves the $w s F$-differentiability of $f$ at $\bar{x}=0$. If we assume that $f$ is $G$-differentiable at this point, then clearly $\nabla_{G} f(0)=0$. Since $\psi \neq$ const, we find $x_{0} \in(0,1)$ with $\psi\left(x_{0}\right) \neq 0$ and put $x_{k}:=2^{-k} x_{0}+2^{-k}$. Then $x_{k} \rightarrow 0$ as $k \rightarrow \infty$ and $\left\|f\left(x_{k}\right)-f(0)\right\| / x_{k}=\left\|\psi_{k}\left(x_{k}\right) e_{k}\right\| / x_{k}=\left|\psi\left(x_{0}\right)\right| /\left(x_{0}+1\right)$ for all $k \in \mathbb{N}$, which contradicts the $G$-differentiability of $f$ at $\bar{x}=0$.

Although the differentiability properties introduced may be weaker than the classical notions in (1), they still imply Lipschitzian behavior in the case of Hadamard and stronger bornologies. We say that $f$ is Lipschitz continuous at $\bar{x}$ if there are $L>0$ and a neighborhood $U$ of $\bar{x}$ such that $\|f(x)-f(\bar{x})\| \leq L\|x-\bar{x}\|$ for all $x \in U$.

Proposition 2.3. The following hold for $\beta \geq H$ :

(i) If $f$ is $w \beta$-differentiable at $\bar{x}$, then it is Lipschitz continuous at $\bar{x}$.

(ii) If $f$ is ws $\beta$-differentiable at $\bar{x}$, then it is Lipschitz continuous around $\bar{x}$.

Proof. It is sufficient to justify (i) for $\beta=H$; the proof of (ii) is similar (cf. also the proof of [1, Prop. 2.2.1]). Assume that $f$ is not Lipschitz at $\bar{x}$. Then for each $k \in \mathbb{N}$ there is $x_{k}$ such that $\left\|x_{k}-\bar{x}\right\| \leq k^{-1}$ and $\left\|f\left(x_{k}\right)-f(\bar{x})\right\| \geq k$. Putting $t_{k}:=\sqrt{k}\left\|x_{k}-\bar{x}\right\|$ and $v_{k}:=\left(x_{k}-\bar{x}\right) / t_{k}$, one has $t_{k} \downarrow 0,\left\|v_{k}\right\|=1 / \sqrt{k}$, and $x_{k}=\bar{x}+t_{k} v_{k}$. Now consider a compact set $V:=\left\{v_{k} \mid k \in \mathbb{N}\right\} \cup\{0\}$ and employ the $w H$-differentiability property of $f$ at $\bar{x}$. For any $y^{*} \in Y^{*}, \varepsilon>0$, and large $k$ we have

$$
\left|\left\langle y^{*},\left[f\left(\bar{x}+t_{k} v\right)-f(\bar{x})\right] / t_{k}\right\rangle-\left[\nabla_{H}\left\langle y^{*}, f\right\rangle(\bar{x})\right] v\right| \leq \varepsilon
$$

whenever $v \in V$. This implies

$$
\left|\left\langle y^{*},\left[f\left(\bar{x}+t_{k} v_{k}\right)-f(\bar{x})\right] / t_{k}\right\rangle\right| \leq\left\|\nabla_{H}\left\langle y^{*}, f\right\rangle(\bar{x})\right\| \cdot\left\|v_{k}\right\|+\varepsilon .
$$

Therefore, the sequence $\left\{\left(f\left(\bar{x}+t_{k} v_{k}\right)-f(\bar{x})\right) / t_{k}\right\}$ weakly converges to 0 and hence is bounded. On the other hand, $\left\|\left(f\left(\bar{x}+t_{k} v_{k}\right)-f(\bar{x})\right) / t_{k}\right\| \geq \sqrt{k} \rightarrow \infty$ as $k \rightarrow \infty$, which is a contradiction.

Note that the $w \beta$-differentiability (ws $\beta$-differentiability) of $f$ at $\bar{x}$ does not automatically imply the existence of the $w \beta$-derivative (resp. $w s \beta$-derivative) in Definition 2.1. However, we can always define the corresponding linear coderivative operator from $Y^{*}$ into $X^{*}$ by

$$
\nabla_{\beta}^{*} f(\bar{x}) y^{*}:=\nabla_{\beta}\left\langle y^{*}, f\right\rangle(\bar{x}) \quad\left(\operatorname{resp} . \nabla_{s \beta}^{*} f(\bar{x}) y^{*}:=\nabla_{s \beta}\left\langle y^{*}, f\right\rangle(\bar{x})\right) .
$$

It follows from the definitions that the operators in (3) are bounded for any $\beta$ if $f$ is Lipschitz continuous at $\bar{x}$, in particular, for the case of $\beta \geq H$ due to Proposition 2.3(i). Using these coderivatives, we obtain conditions ensuring the existence of the corresponding derivatives in Definition 2.1. 
Proposition 2.4. Let $f: X \rightarrow Y$ be $w \beta$-differentiable (resp. ws $\beta$-differentiable) at $\bar{x}$. Then it admits a w $\beta$-derivative (resp. ws $\beta$-derivative) at this point if one of the following conditions holds:

(a) $Y$ is reflexive and $f$ is Lipschitz continuous at $\bar{x}$ (in particular, if $\beta \geq H$ ).

(b) $f$ is directionally differentiable at $\bar{x}$ with respect to the weak topology on $Y$.

Proof. Let us prove (a) for the $w \beta$-case; the $w s \beta$-case is similar. Consider the adjoint coderivative operator $\nabla_{w \beta}^{*} f(\bar{x})^{*}: X^{* *} \rightarrow Y^{* *}=Y$ and define $A:=\left.\nabla_{w \beta}^{*} f(\bar{x})^{*}\right|_{X}$ taking into account that $X \subset X^{* *}$. Then $A: X \rightarrow Y$ is a bounded linear operator, and we can directly check that $A$ satisfies (2) with $x=\bar{x}$. The proof of (b) follows from the definitions.

\section{GRAPHiCAL CODERIVATIVES AND SCALARIZATION}

In contrast to Section 2, this section is devoted to constructions and results of generalized differentiation that are also needed for establishing the main results in Section 4.

Given $\Omega \subset X$ and $\varepsilon \geq 0$, we define the set of $\varepsilon$-normals to $\Omega$ at $\bar{x} \in \Omega$ by

$$
\hat{N}_{\varepsilon}(\bar{x} ; \Omega):=\left\{x^{*} \in X^{*} \mid \limsup _{x \rightrightarrows \bar{x}} \frac{\left\langle x^{*}, x-\bar{x}\right\rangle}{\|x-\bar{x}\|} \leq \varepsilon\right\},
$$

where $x \stackrel{\Omega}{\rightarrow} \bar{x}$ means that $x \rightarrow \bar{x}$ with $x \in \Omega$. The set $\hat{N}(\bar{x} ; \Omega):=\hat{N}_{0}(\bar{x} ; \Omega)$ is a cone called the Fréchet normal cone to $\Omega$ at $\bar{x}$.

Let $\tau=\tau_{X^{*}}$ be an arbitrary topology on the dual space $X^{*}$ that is compatible with the linear structure and satisfies $w^{*} \leq \tau \leq \tau_{\|\cdot\|}$, i.e., it is weaker than or equal to the norm topology $\tau_{\|\cdot\|}$ on $X^{*}$ and is stronger than or equal to the weak-star topology $w^{*}$ on $X^{*}$. Besides $\tau=w^{*}$ and $\tau=\tau_{\|\cdot\|}$, a valuable choice of such a topology is the weak topology on $X^{*}$. The usage of $\tau$ in what follows allows us, first of all, to unify various results for these three major cases. Note that the most interesting results obtained below involve either the weak* topology or the norm topology on one of the spaces in Cartesian products.

Given a topology $\tau$ on $X^{*}$, we define the $\tau$-limiting normal cone to $\Omega \subset X$ at $\bar{x} \in \Omega$ by

$$
N_{\tau}(\bar{x} ; \Omega):=\left\{x^{*} \in X^{*} \mid \exists \varepsilon_{k} \downarrow 0, x_{k} \stackrel{\Omega}{\rightarrow} \bar{x}, x_{k}^{*} \stackrel{\tau}{\rightarrow} x^{*} \text { with } x_{k}^{*} \in \hat{N}_{\varepsilon_{k}}\left(x_{k} ; \Omega\right)\right\} .
$$

The corresponding $\varepsilon$-subdifferential and $\tau$-limiting subdifferential of an extendedreal-valued function $\varphi: X \rightarrow(-\infty, \infty]$ at $\bar{x} \in \operatorname{dom} \varphi$ are defined by

$$
\hat{\partial}_{\varepsilon} \varphi(\bar{x}):=\left\{x^{*} \in X^{*} \mid \liminf _{x \rightarrow \bar{x}} \frac{\varphi(x)-\varphi(\bar{x})-\left\langle x^{*}, x-\bar{x}\right\rangle}{\|x-\bar{x}\|} \geq-\varepsilon\right\}
$$

$\left(\hat{\partial} \varphi(\bar{x}):=\hat{\partial}_{0} \varphi(\bar{x})\right)$ and

$$
\begin{gathered}
\partial_{\tau} \varphi(\bar{x}):=\left\{x^{*} \in X^{*} \mid \exists \varepsilon_{k} \downarrow 0, x_{k} \rightarrow \bar{x}, \varphi\left(x_{k}\right) \rightarrow \varphi(\bar{x}),\right. \\
\left.x_{k}^{*} \stackrel{\tau}{\rightarrow} x^{*} \text { with } x_{k}^{*} \in \hat{\partial}_{\varepsilon_{k}} \varphi\left(x_{k}\right)\right\} .
\end{gathered}
$$

Clearly, the stronger $\tau$ is, the smaller $N_{\tau}(\bar{x} ; \Omega)$ and $\partial_{\tau} \varphi(\bar{x})$ are. For $\tau=w^{*}$ the normal cone and subdifferential reduce to the basic normal cone and subdifferential studied in [7] and are denoted by $N$ and $\partial$ in what follows. Similar to [7, Theorem 2.9], one can equivalently put $\varepsilon_{k}=0$ in (4) and (5) if $\Omega$ is locally closed around $\bar{x}$, 
$\varphi$ is lower semicontinuous around $\bar{x}$, and the space $X$ is Asplund, i.e., every convex continuous function on $X$ is generically Fréchet differentiable.

Given a set-valued mapping $S: X \rightrightarrows Y$ and a topology $\tau=\tau_{X^{*}} \times \tau_{Y^{*}}$ on $X^{*} \times Y^{*}$, we define the $\tau$-limiting coderivative $D_{\tau}^{*} S(\bar{x}, \bar{y}): Y^{*} \rightrightarrows X^{*}$ of $S$ at $(\bar{x}, \bar{y}) \in \operatorname{gph} S$ by

$$
D_{\tau}^{*} S(\bar{x}, \bar{y})\left(y^{*}\right):=\left\{x^{*} \in X^{*} \mid\left(x^{*},-y^{*}\right) \in N_{\tau}((\bar{x}, \bar{y}) ; \operatorname{gph} S)\right\},
$$

where $\bar{y}$ is omitted if the mapping is single-valued at $\bar{x}$. Note that the topologies $\tau_{X^{*}}$ and $\tau_{Y^{*}}$ are of generally different types. In the cases of $\tau=w^{*} \times w^{*}$ and $\tau=w^{*} \times \tau_{\|\cdot\|}$ the coderivative (6) is known as the "normal coderivative" and "mixed coderivative", respectively; see [6] for more details.

Let us establish relationships between $\tau$-coderivatives (6) of $f: X \rightarrow Y$ and the corresponding subdifferentials (5) of its scalarization $\left\langle y^{*}, f\right\rangle$. We need the following condition.

Definition 3.1. Given $f: X \rightarrow Y$ and a topology $\tau=\tau_{Y^{*}}$ on $Y^{*}$, we say that $f$ satisfies the $\tau$-CONVERGENCE CONDITION at $\bar{x}$ if for every $h \in X$ and every sequence $x_{k} \rightarrow \bar{x}, t_{k} \downarrow 0, y_{k}^{*} \stackrel{\tau}{\rightarrow} 0$ one has $\left\langle y_{k}^{*},\left[f\left(x_{k}+t_{k} h\right)-f\left(x_{k}\right)\right] / t_{k}\right\rangle \rightarrow 0$ as $k \rightarrow \infty$.

The $\tau$-convergence condition clearly holds if $\tau=\tau_{\|\cdot\|}$ on $Y^{*}$ (in particular, when $\operatorname{dim} Y<\infty)$ and $f$ is Lipschitzian around $\bar{x}$. For $\tau=w^{*}$ it is always implied by the strict Lipschitzian property of $f$ around $\bar{x}$ in the sense of [7, i.e., if $f$ is Lipschitzian around $\bar{x}$ and the sequence $\left\{\left[f\left(x_{k}+t_{k} h\right)-f\left(x_{k}\right)\right] / t_{k}\right\}$ admits a norm convergent subsequence as $x_{k} \rightarrow \bar{x}, t_{k} \downarrow 0$, and $h \in X$. It is proved by Thibault [12. Theorem 2.3] that the latter property is actually equivalent to the basic version of his original concept of compactly Lipschitzian mappings 11. Let us show that the $w^{*}$-convergence condition is equivalent to the strict Lipschitzian property under an additional assumption on $Y$.

Proposition 3.2. Let $f: X \rightarrow Y$, where $\mathbb{B}_{Y^{*}}$ is $w^{*}$-sequentially compact. Then $f$ satisfies the $w^{*}$-convergence condition at $\bar{x}$ if and only if the above sequence $y_{k}:=\left\{\left[f\left(x_{k}+t_{k} h\right)-f\left(x_{k}\right)\right] / t_{k}\right\}$ admits a norm convergent subsequence for any $h \in X, x_{k} \rightarrow \bar{x}$, and $t_{k} \downarrow 0$ as $k \rightarrow \infty$.

Proof. We need to justify the "only if" part. It follows from the $w^{*}$-convergence condition that $\left\{y_{k}\right\}$ is bounded. Let us show that $\left\{y_{k}\right\}$ is actually totally bounded, which is equivalent to its sequential compactness; see [2, p. 22]. Assuming the contrary, we find $r>0$ such that $\left\{y_{k}\right\} \not \subset Z+r \mathbb{B}_{Y}$ for any finite-dimensional subspace $Z \subset Y$. This allows us to construct a subsequence $\left\{z_{n}\right\}$ of $\left\{y_{k}\right\}$ with $z_{n+1} \notin \operatorname{span}\left\{z_{1}, \ldots, z_{n}\right\}+r \mathbb{B}_{Y}$ for all $n \in \mathbb{N}$. Then we can choose $y_{n}^{*} \in \mathbb{B}_{Y^{*}}$ such that $\operatorname{span}\left\{z_{1}, \ldots, z_{n}\right\} \subset \operatorname{ker}\left(y_{n}^{*}\right)$ and $\left\langle y_{n}^{*}, z_{n+1}\right\rangle \geq r$ for all $n \in \mathbb{N}$. By the assumption, $\left\{y_{n}^{*}\right\}$ contains a subsequence $\left\{y_{n_{m}}^{*}\right\}$ that $w^{*}$-converges to some $y^{*} \in$ $Y^{*}$. We have $\left\langle y^{*}, z_{n}\right\rangle=0$ for all $n \in \mathbb{N}$ by the construction. Hence $\left\langle y_{n_{m}}^{*}-\right.$ $\left.y^{*}, z_{n_{m}+1}\right\rangle=\left\langle y_{n_{m}}^{*}, z_{n_{m}+1}\right\rangle \geq r>0$ for all $m \in \mathbb{N}$, which contradicts the $w^{*}$ convergence condition.

Theorem 3.3. Let $f: X \rightarrow Y$ with $\tau=\tau_{X^{*}} \times \tau_{Y^{*}}$ on $X^{*} \times Y^{*}$. The following hold for all $y^{*} \in Y^{*}$ :

(i) If $f$ is continuous around $\bar{x}$, then $\partial_{\tau_{X^{*}}}\left\langle y^{*}, f\right\rangle(\bar{x}) \subset D_{\tau}^{*} f(\bar{x})\left(y^{*}\right)$.

(ii) If $f$ is Lipschitz continuous around $\bar{x}$ and $\tau=\tau_{X^{*}} \times \tau_{\|\cdot\|}$, then $D_{\tau}^{*} f(\bar{x})\left(y^{*}\right)=$ $\partial_{\tau_{X^{*}}}\left\langle y^{*}, f\right\rangle(\bar{x})$. 
(iii) Let $X$ be Asplund, let $\tau=w^{*} \times \tau_{Y^{*}}$, and let $f$ be Lipschitz continuous around

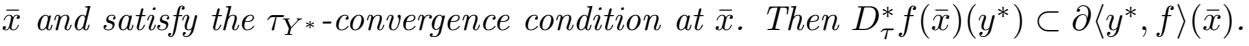

Proof. Assertion (i) follows from the definitions; cf. the proof of [7, Theorem 5.2]. To prove (ii), we first consider an arbitrary topology $\tau$ on $X^{*} \times Y^{*}$. Pick any $x^{*} \in D_{\tau}^{*} f(\bar{x})\left(y^{*}\right)$ and find $\varepsilon_{k} \downarrow 0, x_{k} \rightarrow \bar{x},\left(x_{k}^{*}, y_{k}^{*}\right) \stackrel{\tau}{\rightarrow}\left(x^{*}, y^{*}\right)$ such that $\left(x_{k}^{*},-y_{k}^{*}\right) \in$ $\hat{N}_{\varepsilon_{k}}\left(\left(x_{k}, f\left(x_{k}\right)\right)\right.$; gph $\left.f\right)$ for all $k \in \mathbb{N}$. Hence

(7) $\left\langle x_{k}^{*}, x-x_{k}\right\rangle-\left\langle y_{k}^{*}, f(x)-f\left(x_{k}\right)\right\rangle \leq 2 \varepsilon_{k}(1+L)\left\|x-x_{k}\right\|$ for all $x \in x_{k}+\eta_{k} \mathbb{B}_{X}$

with some $\eta_{k} \downarrow 0$, where $L$ is a Lipschitz constant of $f$ around $\bar{x}$. The latter yields $x_{k}^{*} \in \hat{\partial}_{\tilde{\varepsilon}_{k}}\left\langle y^{*}, f\right\rangle\left(x_{k}\right)$ with $\tilde{\varepsilon}_{k}:=2 \varepsilon_{k}(1+L)+L\left\|y_{k}^{*}-y^{*}\right\|$. When $\tau_{Y^{*}}=\|\cdot\|$, we have $\tilde{\varepsilon}_{k} \downarrow 0$ and thus $x^{*} \in \partial_{\tau_{X^{*}}}\left\langle y^{*}, f\right\rangle(\bar{x})$, which gives (ii). To justify (iii), we follow the proof of [7, Theorem 5.2] using (7) and the "fuzzy sum rule" of [3, Theorem 2]. In this way we get $x^{*}=x_{1}^{*}+x_{2}^{*}$ with $x_{1}^{*} \in \partial\left\langle y^{*}, f\right\rangle(\bar{x})$. The $\tau_{Y^{*} \text {-convergence condition }}$ allows us to conclude that $x_{2}^{*}=0$.

Corollary 3.4. Let $f: X \rightarrow Y$ be locally Lipschitzian around $\bar{x}$, let $\tau=w^{*} \times \tau_{Y^{*}}$, and let $\tilde{\tau}=\tau_{X^{*}} \times \tilde{\tau}_{Y^{*}}$ with $\tau_{Y^{*}} \leq \tilde{\tau}_{Y^{*}} \leq \tau_{\|\cdot\|}$. Assume that $X$ is Asplund and that

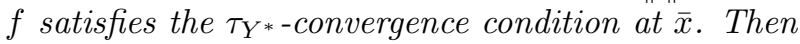

$$
D_{\tau}^{*} f(\bar{x})\left(y^{*}\right)=D_{\tilde{\tau}}^{*} f(\bar{x})\left(y^{*}\right)=\partial\left\langle y^{*}, f\right\rangle(\bar{x}) \text { for all } y^{*} \in Y^{*} \text {. }
$$

\section{Subspace PROPERTY AND GRAPHICAL REGUlARITY}

In this section we apply constructions of Sections 2 and 3 to obtain various infinite-dimensional counterparts and extensions of Rockafellar's results mentioned in the Introduction. Our proofs are simple and different from those in [9] even in finite dimensions.

Given $\Omega \subset X$ and $\tau$ on $X^{*}$, we consider the normal cone (4) and its closed convexification

$$
\bar{N}_{\tau}(\bar{x} ; \Omega):=\mathrm{cl}^{*} \operatorname{co} N_{\tau}(\bar{x} ; \Omega),
$$

where $\mathrm{cl}^{*}$ stands for the weak ${ }^{*}$ topological closure in $X^{*}$. Clearly, (8) depends on $\tau$. It is proved in 7 , Theorem 8.1] that, for closed $\Omega$ in Asplund spaces and for $\tau=w^{*}$, the cone (8) agrees with Clarke's normal cone defined [1] as the polar to his tangent cone in any Banach spaces.

Theorem 4.1. Let $X$ and $Y$ be Banach with $\tau=w^{*} \times \tau_{Y^{*}}$. The following are equivalent:

(a) For every $f: X \rightarrow Y$, which is Lipschitz continuous around some point $\bar{x} \in X$

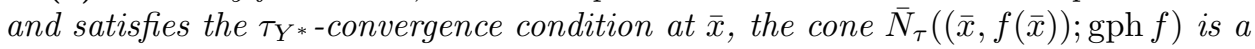
linear subspace of $X^{*} \times Y^{*}$.

(b) $X$ is an Asplund space.

Proof. First we prove (b) $\Rightarrow$ (a) based on Theorem 3.3 and the following two facts held for locally Lipschitzian functions $\varphi: X \rightarrow \mathbb{R}$ :

(i) $\partial_{C}(-\varphi)(\bar{x})=-\partial_{C} \varphi(\bar{x})$ for Clarke's generalized gradient when $X$ is Banach 1, Prop. 2.3.1].

(ii) $\partial_{C} \varphi(\bar{x})=\mathrm{cl}^{*} \operatorname{co} \partial \varphi(\bar{x})$ when $X$ is Asplund [7, Theorem 8.11].

Now taking $\left(x^{*},-y^{*}\right) \in N_{\tau}((\bar{x}, f(\bar{x}))$; gph $f)$, we get

$$
\begin{aligned}
x^{*} & \in D_{\tau}^{*} f(\bar{x})\left(y^{*}\right) \subset \partial\left\langle y^{*}, f\right\rangle(\bar{x}) \subset \partial_{C}\left\langle y^{*}, f\right\rangle(\bar{x})=-\partial_{C}\left\langle-y^{*}, f\right\rangle(\bar{x}) \\
& =-\operatorname{cl}^{*} \operatorname{co} \partial\left\langle-y^{*}, f\right\rangle(\bar{x}) \subset-\mathrm{cl}^{*} \operatorname{co} D_{\tau}^{*} f(\bar{x})\left(-y^{*}\right),
\end{aligned}
$$


which gives $-N_{\tau}((\bar{x}, f(\bar{x}))$; gph $f) \subset \operatorname{cl}^{*} \operatorname{co} N_{\tau}((\bar{x}, f(\bar{x})) ; \operatorname{gph} f)$ and the subspace property.

To prove $(\mathrm{a}) \Rightarrow(\mathrm{b})$, we consider an arbitrary convex function $\psi: X \rightarrow \mathbb{R}$ continuous around $\bar{x} \in X$. Given $Y$, we represent it as $Y=\mathbb{R} \times Y_{1}$, where $Y_{1}$ is a subspace of $Y$, and define a Lipschitzian mapping $f: X \rightarrow Y$ by $f(x):=(\psi(x), 0)$. It is easy

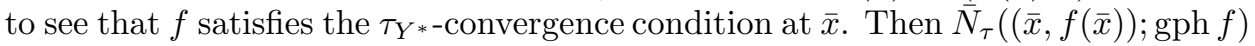
is a subspace of $X^{*} \times Y^{*}$. Since

$$
\operatorname{gph} f=\operatorname{gph} \psi \times\{0\} \text { and } N_{\tau}((\bar{x}, f(\bar{x})) ; \operatorname{gph} f)=N((\bar{x}, \psi(\bar{x})) ; \operatorname{gph} \psi) \times Y_{1}^{*},
$$

it follows that $\bar{N}((\bar{x}, \psi(\bar{x})) ; \operatorname{gph} \psi)$ is a subspace of $X^{*} \times \mathbb{R}$. Due to the convexity and continuity of $\psi$ we have $\partial \psi(\bar{x}) \neq \emptyset$ and

$$
N((\bar{x}, \psi(\bar{x})) ; \operatorname{gph} \psi)=\left\{\left(x^{*},-\lambda\right) \mid x^{*} \in \partial(\lambda \psi)(\bar{x}), \lambda \in \mathbb{R}\right\}
$$

(the latter holds for any locally Lipschitzian function). Thus $\partial(-\psi)(\bar{x}) \neq \emptyset$; otherwise we get a contradiction with the subspace property of $\bar{N}((\bar{x}, \psi(\bar{x}))$; gph $\psi)$. Since $\psi$ was chosen arbitrarily, one has $\partial \varphi(\bar{x}) \neq \emptyset$ for any concave continuous function $\varphi: X \rightarrow \mathbb{R}$ at every $\bar{x} \in X$. By (5) with $\tau=w^{*}$ this ensures that the set $\left\{x \in X \mid \hat{\partial}_{\varepsilon} \varphi(x) \neq \emptyset\right\}$ is dense in $X$ for every $\varepsilon>0$, which implies the Asplund property of $X$ due to [4, Prop. 1].

Remark 4.2. The proof of Theorem 4.1 allows us to justify the subspace property of Clarke's normal cone to the graph of every compactly Lipschitzian mapping $f: X \rightarrow Y$ between arbitrary Banach spaces. Indeed, it follows from the above arguments due to the scalarization formula for Ioffe's approximate coderivative established in [5, Theorem 4]. After receiving a preprint of our paper, L. Thibault (personal communication) found another proof of the latter subspace property, in the case of any Lipschitzian mapping between normed spaces, based on a sequential characterization of Clarke's tangent cone.

Next let us establish relationships between the single-valuedness of graphical coderivatives ([6) and the $w s \beta$-differentiability properties of $f$ in the most interesting cases of $\beta=F, H$. In this way we obtain conditions under which the graphical coderivatives agree with the corresponding linear coderivatives in (31).

Theorem 4.3. Let $f: X \rightarrow Y$ with $\tau=\tau_{X^{*}} \times \tau_{Y^{*}}$ on $X^{*} \times Y^{*}$. The following hold:

(i) Assume that $f$ is wsF-differentiable at $\bar{x}$ and that $\tau_{Y^{*}}=\tau_{\|\cdot\| \cdot}$. Then $D_{\tau}^{*} f(\bar{x})$ is a single-valued bounded linear operator equal to $\nabla_{s F}^{*} f(\bar{x})$ for any $\tau_{X^{*}}$.

(ii) Assume that $f$ is wsH-differentiable and satisfies the $\tau_{Y^{*}-\text {-convergence con- }}$ dition at $\bar{x}$, that $X$ is Asplund, and that $\tau_{X^{*}}=w^{*}$. Then $D_{\tau}^{*} f(\bar{x})$ is a single-valued bounded linear operator equal to $\nabla_{s H}^{*} f(\bar{x})$.

(iii) Assume that $f$ is Lipschitz continuous around $\bar{x}$, that $X$ is Asplund, and that $D_{\tau}^{*} f(\bar{x})$ is single-valued for $\tau=w^{*} \times \tau_{Y^{*}}$. Then $f$ is wsH-differentiable at $\bar{x}$ and $D_{\tau}^{*} f(\bar{x})$ is a bounded linear operator equal to $\nabla_{s H}^{*} f(\bar{x})$.

Proof. (i) One can check that $\partial_{\tau_{X^{*}}}\left\langle y^{*}, f\right\rangle(\bar{x})=\nabla_{s F}^{*} f(\bar{x}) y^{*}$ for all $y^{*} \in Y^{*}$ if $f$ is $w s F$-differentiable at $\bar{x}$. Thus (i) follows from Theorem [3.3(ii).

To prove (ii), we use Corollary 3.4 which gives $D_{\tau}^{*} f(\bar{x})\left(y^{*}\right)=\partial\left\langle y^{*}, f\right\rangle(\bar{x})$. Then (ii) follows from $\partial_{C}\left\langle y^{*}, f\right\rangle(\bar{x})=\left\{\nabla_{s H}\left\langle y^{*}, f\right\rangle(\bar{x})\right\}$ if $\left\langle y^{*}, f\right\rangle$ is $s H$-differentiable (see [1, Prop. 2.2.1]) and from $\partial\left\langle y^{*}, f\right\rangle(\bar{x}) \neq \emptyset$ if $X$ is Asplund (see [7, Cor. 3.9]). 
The converse statement (iii) follows from Theorem [3.3(i) and assertion (ii) in the proof of Theorem 4.1, which ensure that $\emptyset \neq \partial\left\langle y^{*}, f\right\rangle(\bar{x})=\partial_{C}\left\langle y^{*}, f\right\rangle(\bar{x})$ is a singleton, and hence $\left\langle y^{*}, f\right\rangle$ is $s H$-differentiable at $\bar{x}$ due to [1, Prop. 2.2.1].

Corollary 4.4. Let $f: X \rightarrow \mathbb{R}^{m}$ be Lipschitz continuous around $\bar{x}$, and let $X$ be Asplund with $\tau_{X^{*}}=w^{*}$. The following are equivalent:

(i) Clarke's normal cone to gph $f$ at $(\bar{x}, f(\bar{x}))$ is a linear subspace of dimension $m$.

(ii) The basic normal cone $N((\bar{x}, f(\bar{x}))$; gph $f)$ is a linear subspace of dimension $m$.

(iii) $f$ is strictly Hadamard differentiable at $\bar{x}$.

Proof. Equivalence (ii) $\Leftrightarrow$ (iii) follows from Theorem 4.3, since the $\tau_{Y^{*} \text {-convergence }}$ condition automatically holds for $Y=\mathbb{R}^{m}$, and due to the fact that the graph of any bounded linear operator is isomorphic to the domain space. Equivalence (i) $\Leftrightarrow($ ii) follows from the representation of Clarke's normal cone in form (86) under the assumptions made.

For $X=\mathbb{R}^{n}$ Hadamard strict differentiability reduces to the usual (Fréchet) strict differentiability, in which case the equivalence (i) $\Leftrightarrow$ (iii) in Corollary 4.4 is proved in a different way by Rockafellar [9. Theorem 3.5]. Taking into account Remark 4.2 and the proof of Theorem 4.3 the latter equivalence can be established for arbitrary Banach spaces $X$.

Finally, let us obtain relationships between graphical regularity of Lipschitzian mappings and the differentiability properties considered in Section 2. We say that $f: X \rightarrow Y$ is graphically $\tau$-regular at $\bar{x}$ if

$$
N_{\tau}((\bar{x}, f(\bar{x})) ; \operatorname{gph} f)=\hat{N}((\bar{x}, f(\bar{x})) ; \operatorname{gph} f) .
$$

Theorem 4.5. Let $f: X \rightarrow Y$ with $\tau=\tau_{X^{*}} \times \tau_{Y^{*}}$ on $X^{*} \times Y^{*}$. The following hold:

(i) If $f$ is wsF-differentiable at $\bar{x}$, then it is graphically $\tau$-regular at $\bar{x}$ for $\tau=$ $\tau_{X^{*}} \times \tau_{\|\cdot\|}$ with any $\tau_{X^{*}}$.

(ii) Assume that $X$ is Asplund, that $\tau=w^{*} \times \tau_{Y^{*}}$, and that $f$ satisfies the

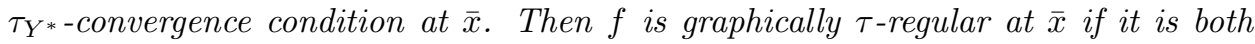
$w F$-differentiable and wsH-differentiable at this point.

(iii) Conversely, for any $\tau_{Y^{*}}$, the graphical $w^{*} \times \tau_{Y^{*}}$-regularity of $f$ at $\bar{x}$ implies both $w F$-differentiability and wsH-differentiability of $f$ at this point provided that $X$ is Asplund and that $f$ is Lipschitz continuous around $\bar{x}$.

Proof. Assertion (i) follows from Theorem 4.3(i) and the formula

$$
\hat{\partial}\left\langle y^{*}, f\right\rangle(\bar{x})=\hat{D}^{*} f(\bar{x})\left(y^{*}\right):=\left\{x^{*} \in X^{*} \mid\left(x^{*},-y^{*}\right) \in \hat{N}((\bar{x}, f(\bar{x})) ; \operatorname{gph} f)\right\}
$$

that can be easily justified for any $f$ Lipschitz continuous around $\bar{x}$.

To prove (ii), we observe that $\partial\left\langle y^{*}, f\right\rangle(\bar{x})$ is a singleton under the assumptions made in Theorem 4.3(ii). On the other hand, the $w F$-differentiability of $f$ at $\bar{x}$ gives $\hat{\partial}\left\langle y^{*}, f\right\rangle(\bar{x})=\left\{\nabla_{F}\left\langle y^{*}, f\right\rangle(\bar{x})\right\}$. Combining this with (9), we get

$$
D_{\tau}^{*} f(\bar{x})\left(y^{*}\right)=\partial\left\langle y^{*}, f\right\rangle(\bar{x})=\hat{\partial}\left\langle y^{*}, f\right\rangle(\bar{x})=\hat{D}^{*} f(\bar{x})\left(y^{*}\right) \text { for all } y^{*} \in Y^{*},
$$

which justifies the $\tau$-regularity of $f$ at $\bar{x}$. 
It remains to prove (iii). As mentioned above, $\partial\left\langle y^{*}, f\right\rangle(\bar{x}) \neq \emptyset$ for all $y^{*} \in Y^{*}$ when $X$ is Asplund. Picking any $x^{*} \in \partial\left\langle y^{*}, f\right\rangle(\bar{x})$, we have $x^{*} \in D_{\tau}^{*} f(\bar{x})\left(y^{*}\right)$ by Theorem 3.3(i), and hence

$$
\left(x^{*},-y^{*}\right) \in N_{\tau}((\bar{x}, f(\bar{x})) ; \operatorname{gph} f)=\hat{N}((\bar{x}, f(\bar{x})) ; \operatorname{gph} f)
$$

due to the assumed $\tau$-regularity. Using (9), we get

$$
\hat{\partial}\left\langle y^{*}, f\right\rangle(\bar{x})=\partial\left\langle y^{*}, f\right\rangle(\bar{x}) \neq \emptyset \text { for all } y^{*} \in Y^{*},
$$

which clearly implies the Fréchet differentiability of $\left\langle y^{*}, f\right\rangle$ at $\bar{x}$. Therefore

$$
\partial_{C}\left\langle y^{*}, f\right\rangle(\bar{x})=\mathrm{cl}^{*} \operatorname{co} \partial\left\langle y^{*}, f\right\rangle(\bar{x})=\left\{\nabla_{F}\left\langle y^{*}, f\right\rangle(\bar{x})\right\} .
$$

So $\left\langle y^{*}, f\right\rangle$ must be $s H$-differentiable at $\bar{x}$ for any $y^{*} \in Y^{*}$, since $\partial_{C}\left\langle y^{*}, f\right\rangle(\bar{x})$ is a singleton.

Corollary 4.6. Let $f: X \rightarrow \mathbb{R}^{m}$ be Lipschitz continuous around $\bar{x}$, and let $X$ be Asplund with $\tau_{X^{*}}=w^{*}$. Then the following are equivalent:

(i) $f$ is graphically regular at $\bar{x}$.

(ii) $f$ is simultaneously Fréchet differentiable and strictly Hadamard differentiable at $\bar{x}$.

\section{LIPSCHITZIAN SETS AND SET-VALUED MAPPINGS}

Although the results of Section 4 concern single-valued mappings, they can be used for the study of sets and set-valued mappings generated by graphs of singlevalued Lipschitzian mappings via smooth transformations. Rockafellar [9] (see also 10]) considered such "Lipschitzian manifolds" and "graphically Lipschitzian mappings" in finite dimensions and showed that they include maximal monotone operators and subdifferential mappings for important classes of functions typically encountered in optimization and variational analysis. Let us now demonstrate that the results obtained above can be extended to (appropriately defined) Lipschitztype sets and set-valued mappings in infinite-dimensions with the help of normal cone calculus established in [8]. Since set-valued mappings reduce to their graphs, it is sufficient to formulate results only in the case of sets. For simplicity we confine our consideration to the basic $\tau=w^{*}$ topology.

Definition 5.1. (i) A subset $\Omega$ of a Banach space $Z$ is HEMI-LIPSCHITZIAN around $\bar{z} \in \Omega$ if there are a mapping $f: X \rightarrow Y$ between Banach spaces and a mapping $g: Z \rightarrow X \times Y$ with $g(\bar{z})=(\bar{x}, f(\bar{x}))$ such that $g$ is strictly $F$-differentiable at $\bar{z}$ with the surjective derivative, that $f$ is Lipschitz continuous around $\bar{x}$, and that

$$
\Omega \cap U=g^{-1}(V \cap \operatorname{gph} f)
$$

for some neighborhoods $U$ of $\bar{z}$ and $V$ of $g(\bar{z})$. We say that $\Omega$ is STRICTLY HEMILIPSCHITZIAN around $\bar{z}$ if, in addition, $f$ satisfies the $w^{*}$-convergence condition at $\bar{x}$.

(ii) Let $\mathcal{B}$ stand for some differentiability concept (e.g., $\mathcal{B}=\beta, w \beta$, ws $\beta$ ). We say that $\Omega$ is $\mathcal{B}$-HEMismoоTH at $\bar{z}$ if it is hemi-Lipschitzian around this point and $f$ can be chosen as $\mathcal{B}$-differentiable at $\bar{x}$.

(iii) A set-valued mapping $S$ between Banach spaces is GRAPHICALLY HEMILIPSCHITZIAN around some point $\bar{z} \in \operatorname{gph} S$ (resp. GRAPHICALLY $\mathcal{B}$-HEMISMOOTH at $\bar{z}$ ) if $\operatorname{gph} S$ is hemi-Lipschitzian around $\bar{z}$ (resp. $\mathcal{B}$-hemismooth at $\bar{z}$ ). 
Rockafellar's notion of "Lipschitzian manifold" [9] reduces to Definition [5.1(i) if $g: \mathbb{R}^{d} \rightarrow \mathbb{R}^{n} \times \mathbb{R}^{m}$ is a locally $\mathcal{C}^{1}$ function with the nonsingular Jacobian at $\bar{z}$; hence $g^{-1}$ is also locally $\mathcal{C}^{1}$. The corresponding set-valued mappings are called graphically Lipschitzian in [10].

Theorem 5.2. Let $\Omega \subset Z$ be strictly hemi-Lipschitzian around $\bar{z}$, where the space $X$ in Definition 5.1(i) can be chosen as Asplund. Then the following hold:

(i) The convexified normal cone $\mathrm{cl}^{*} \operatorname{co} N(\bar{z} ; \Omega)$ is a linear subspace of $Z^{*}$.

(ii) $\Omega$ is normally regular at $\bar{z}$ (that is, $N(\bar{z} ; \Omega)=\hat{N}(\bar{z} ; \Omega)$ ) if and only if it is simultaneously $w F$-smooth and wsH-smooth at $\bar{z}$, i.e., $f$ in Definition 5.1)(ii) has both of these properties at $\bar{x}$.

Proof. Given a mapping $g: Z \rightarrow W$ between Banach spaces, we have the calculus rules

$$
N\left(\bar{z} ; g^{-1}(\Lambda)\right)=\nabla g(\bar{z})^{*} N(g(\bar{z}) ; \Lambda) \text { and } \hat{N}\left(\bar{z} ; g^{-1}(\Lambda)\right)=\nabla g(\bar{z})^{*} \hat{N}(g(\bar{z}) ; \Lambda)
$$

for any subset $\Lambda \subset W$ with $g(\bar{z}) \in \Lambda$ provided that $g$ is strictly $F$-differentiable at $\bar{z}$ with the surjective derivative $\nabla g(\bar{z})$; see [8] for the proof of these and related results involving the Lyusternik-Graves theorem on metric regularity. Then (i) follows directly from Theorem 4.1 and the first formula in (10). To prove (ii), we observe that, for every set $\Omega$ hemi-Lipschitzian around $\bar{x}$, the normal regularity of $\Omega$ at $\bar{z}$ is equivalent to the graphical regularity of $f$ at $\bar{x}$. Indeed, it follows from (10) and the well-known fact that the adjoint operator to any surjective linear bounded operator is injective (one-to-one). Now (ii) is implied by Theorem 4.5 .

It follows from Remark 4.2 and the proof of Theorem 5.2 that the subspace property also holds for Clarke's normal cone to hemi-Lipschitzian sets provided that $f: X \rightarrow Y$ in Definition 5.1 is compactly Lipschitzian and all the spaces involved are arbitrary Banach.

\section{ACKNOWLEDGMENT}

The authors gratefully acknowledge the valuable remarks and suggestions of an anonymous referee that helped to improve the original presentation. In particular, the referee suggested the proof of Proposition 3.2 presented in the paper (our original proof worked in the case of reflexive spaces $Y$ or, at most, under the assumption that both $\mathbb{B}_{Y^{*}}$ and $\mathbb{B}_{Y^{* *}}$ are $w^{*}$-sequentially compact). We are also thankful to Jonathan Borwein, Marian Fabian, Terry Rockafellar, and Lionel Thibault for fruitful discussions and comments.

\section{REFERENCES}

[1] F. H. Clarke, Optimization and Nonsmooth Analysis, Wiley, New York, 1983. MR 85m:49002

[2] N. Dunford and J. T. Schwartz, Linear Operators, Part I, Interscience, New York, 1958. MR 22:8302

[3] M. Fabian, Subdifferentiability and trustworthiness in the light of a new variational principle of Borwein and Preiss, Acta Univ. Carolinae 30 (1989), 51-56. MR 91c:49024

[4] A. D. Ioffe, On subdifferentiability spaces, Ann. New York Acad. Sci. 410 (1983), 107-119. MR 86g:90124

[5] - Directional compactness, scalarization and nonsmooth semi-Fredholm mappings, Nonlinear Anal. 29 (1997), 201-219. MR 98c:49043

[6] B. S. Mordukhovich, Coderivatives of set-valued mappings: calculus and applications, Nonlinear Anal. 30 (1997), 3059-3070. 
[7] B. S. Mordukhovich and Y. Shao, Nonsmooth sequential analysis in Asplund spaces, Trans. Amer. Math. Soc. 348 (1996), 1235-1280. MR 96h:49036

[8] B. S. Mordukhovich and B. Wang, Restrictive metric regularity and generalized differential calculus in Banach spaces, preprint, Department of Mathematics, Wayne State University, 2002.

[9] R. T. Rockafellar, Maximal monotone relations and the second derivatives of nonsmooth functions, Ann. Inst. Henri Poincaré 2 (1985), 167-184. MR 87c:49021

[10] R. T. Rockafellar and R. J.-B. Wets, Variational Analysis, Springer, Berlin, 1998. MR 98m:49001

[11] L. Thibault, Sous-différentiel de fonctions vectorielles compactement lipschitziennes, C. R. Acad. Sc. Paris 286 (1978), 995-998. MR 80b:58014

[12] _ On compactly Lipschitzian mappings, Recent Advances in Optimization (P. Gritzmann et al., eds.), Lecture Notes in Econ. Math. Syst., Ser. 456, pp. 356-364, Springer, Berlin, 1997. MR 98f:49019

Department of Mathematics, Wayne State University, Detroit, Michigan 48202

E-mail address: boris@math. wayne.edu

Department of Mathematics, Eastern Michigan University, Ypsilanti, Michigan 48197

E-mail address: wangbw@math.emich.edu 